BNL - 67511

CAP-286-NuFact-00R

NuMu Note \#129

Informal Report

\title{
MUON POLARIZATION EFFECTS IN THE FRONT \\ END OF THE NEUTRINO FACTORY
}

R.C. Fernow and J.C. Gallardo

Brookhaven National Laboratory, New York

Y. Fukui

Univ. of California, Los Angeles 
NuMu Note \#129

\title{
Muon polarization effects in the Front End of the Neutrino Factory
}

\author{
R.C. Fernow \& J.C. Gallardo \\ Brookhaven National Laboratory \\ Y. Fukui \\ University of California at Los Angeles
}

30 May 2000

\begin{abstract}
We summarize the methods used for simulation of polarization effects in the front end of a possible neutrino factory. We first discuss the helicity of muons in the pion decay process. We find that, neglecting acceptance considerations, the average helicity asymptotically approaches a magnitude of 0.185 at large pion momenta. Next we describe the methods used for tracking the spin through the complicated electromagnetic field configurations in the front end of the neutrino factory, including $r f$ phase rotation and ionization cooling channels. Various depolarizing effects in matter are then considered, including multiple Coulomb scattering and elastic scattering from atomic electrons. Finally, we include all these effects in a simulation of a $480 \mathrm{~m}$ long, double phase rotation front end scenario.
\end{abstract}

\section{Introduction}

A polarized muon beam should provide a valuable handle for understanding the physics at a neutrino factory since the polarization affects the angular distributions of the neutrinos coming from the muon decay [1]. In a similar manner polarization information is also thought to be valuable for understanding the physics in a muon collider [2]. 
Muon beam polarization is normally described in terms of the helicity of each muon, which is defined to be the projection of the spin vector $\mathbf{s}$ in the muon rest frame along its momentum $\mathbf{p}$ or

$$
h=\frac{\vec{p} \cdot \hat{s}}{|\vec{p}|}
$$

The spin vector is normalized to 1 in the muon rest frame. Thus $\mathrm{h}$ is a variable bounded in the range $[-1,1]$. It is well known [3] that the positive muon has helicity $=-1$ in the positive pion rest frame. However, in the transformation of the muon into the $L A B$ frame an angle develops between the transformed muon's momentum and its spin in the rest frame. As a result the magnitude of the helicity in the LAB frame is generally less than 1.

After the muon has been created it must propagate through a maze of complicated electric and magnetic fields in the front end of a neutrino factory or muon collider. Since the spin evolution follows a different set of equations than the momentum, small depolarization effects can be introduced that reduce the average polarization of the muon beam. In addition the cooling channels contain substantial amounts of material in the absorbers, absorber windows, and if cavity windows if pillbox cavities are used. Large angle Coulomb scattering and elastic scattering from atomic electrons have a finite probability of flipping the muon spin direction and can thus lead to further depolarization of the muon beam.

\section{Muon polarization in pion decay}

Hayakawa [4] gives the following expression for the magnitude of the muon helicity in the LAB frame

$$
h=\frac{E E^{*}-\gamma_{\pi} m^{2}}{p p^{*}}
$$

where $\{\mathrm{m}, \mathrm{p}, \mathrm{E}\}$ refer to the muon's \{mass, momentum, energy\}, \{starred, unstarred\} quantities refer to the \{pion rest frame, LAB frame and $\gamma_{\pi}$ is the pion relativistic energy factor in the LAB frame.

An alternative expression for the muon helicity can be derived from the Wigner rotation formalism $[5,6]$

$$
h=\frac{E_{\pi} p^{*}+E^{*} p_{\pi} \cos \theta^{*}}{m_{\pi} p}
$$

where, as before, the unsubscripted variables refer to the muon. 
A third method starts with the known spin vector in the pion rest frame. The spin vector is boosted to the LAB frame using the appropriate Lorentz transformation. A second Lorentz transformation takes the spin vector to the muon rest frame [7]. The helicity can then be calculated directly from the definition in Eq. 1 . This method is described more fully in section 3 .

All three methods give the same value for the helicity.

Figure 1 shows the variation of the average muon helicity in the $L A B$ frame as a function of the pion momentum. The solid curve is from a calculation using the Wigner formalism given in Eq. 3. The points are from Icool [8], which uses the Hayakawa expression given in Eq. 2.

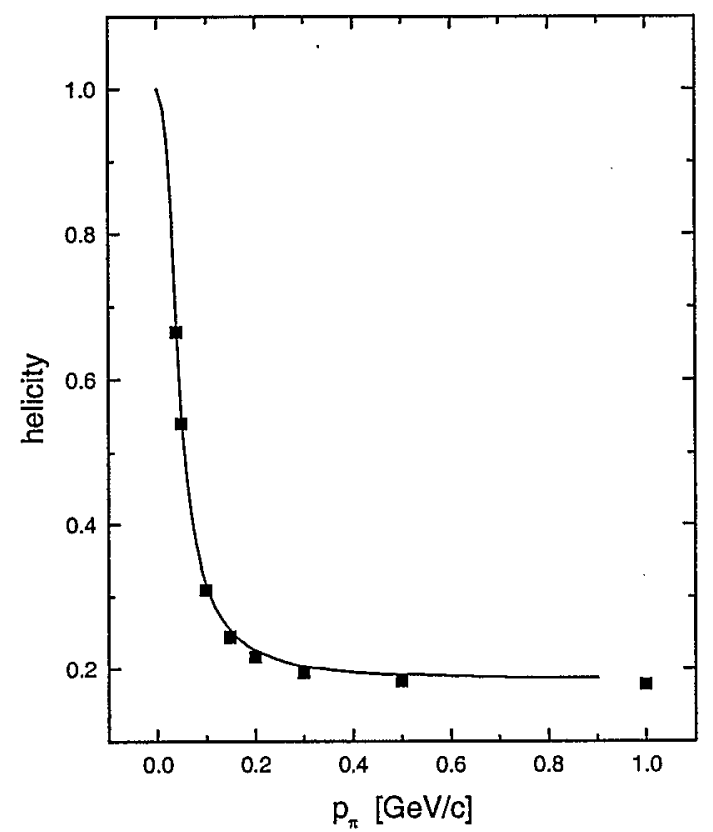

Figure 1. Magnitude of the average muon helicity in the $\mathrm{LAB}$ frame as a function of pion momentum. The squares are Icool simulations using the Hayakawa equation. The solid curve is from an independent simulation using the Wigner formalism.

The Icool simulation used a monochromatic pion beam with 0 transverse emittance. We averaged the helicity for 10000 random pion decays. We see that both simulations agree and that the helicity has the value -1 as it should at very low pion momentum. When the average helicity function shown in Fig. 1 is convoluted with the accepted muon momentum spectrum for typical muon collider scenarios [9], the average polarization of the accepted muon beam is typically 
$\sim 20 \%$. As the pion momentum increases, the helicity falls rapidly and approaches an asymptotic value of $\sim 0.18$. An analytic derivation, given in the appendix of this paper, shows that the magnitude of the asymptotic average helicity of the produced muons is 0.185 .

\section{Spin tracking}

When spin tracking is required each muon needs an associated 3-component, normalized spin vector in the muon rest frame. Let us first describe how this vector is determined. In the pion rest frame, where the helicity is \pm 1 , we know that the spin vector $\mathbf{s}^{*}$ lies along the muon momentum and has the magnitude $\gamma^{*}=1.04$. Using our knowledge of the pion kinematics in the LAB, we transform the spin into the LAB frame [10]

$$
\vec{S}=\frac{E^{*} \hat{p}^{*}+\left(\gamma_{\pi}-1\right) \frac{E^{*}}{\beta_{\pi}^{2}}\left(\hat{p}^{*} \cdot \vec{\beta}_{\pi}\right) \vec{\beta}_{\pi}+\gamma_{\pi} p^{*} \vec{\beta}_{\pi}}{m}
$$

Expressed as a 4-vector, the 0 component of the spin is

$$
S_{0}= \pm \sqrt{|\vec{S}|^{2}-1}
$$

The negative sign is used when

$$
E<\frac{\gamma_{\pi} m^{2}}{E^{*}}
$$

Another expression for the helicity can be found from $S_{0}$

$$
h=\frac{S_{0} m}{p}
$$

Now that we know the spin vector in the LAB frame, we can transform to the muon rest frame using [11]

$$
\vec{s}=\vec{S}-\frac{\gamma}{\gamma+1}(\vec{\beta} \cdot \vec{S}) \vec{\beta}
$$


where $\{\boldsymbol{\beta}, \gamma\}$ refer to the muon in the $L A B$ frame.

The muon spin vector in the rest frame evolves in electric and magnetic fields according to the Thomas-BMT equation $[7,12]$

$$
\frac{d \vec{s}}{d z}=-\frac{e}{p_{z}} \vec{\Omega} \times \vec{s}
$$

where the vector $\boldsymbol{\Omega}$ is given in a non-dipole field region by

$$
\vec{\Omega}=(a \gamma+1) \vec{B}-a(\gamma-1)(\hat{v} \cdot \vec{B}) \hat{v}+\gamma\left(a+\frac{1}{\gamma+1}\right) \vec{E} \times \vec{\beta}
$$

In this equation $\mathbf{E}$ and $\mathbf{B}$ are the fields in the laboratory and $\{\mathbf{v}, \boldsymbol{\beta}, \gamma\}$ refer to the muon in the laboratory frame. The quantity $a$ is the g-factor anomaly for the muon, which has the value $1.16510^{-3}$.

During spin tracking the current value of the vector $\boldsymbol{\Omega}$ is saved at the start of every step. A new value $\mathbf{s}^{\prime}$ of the spin is then computed using Eq. 9 . However, we know from the form of Eq. 9 that the component of the spin along $\mathbf{\Omega}$ must be conserved. To minimize the effects of numerical inaccuracies we recompute the spin vector $\mathbf{s}^{\prime \prime}$ at the end of the step as

$$
\vec{s}^{\prime \prime}=(\vec{s} \cdot \hat{\Omega}) \hat{\Omega}+\sqrt{1-(\vec{s} \cdot \hat{\Omega})^{2}} \frac{\vec{R}}{|\vec{R}|}
$$

where the auxiliary vector $\mathbf{R}$ is given by

$$
\vec{R}=\vec{s}^{\prime}-(\vec{s} \cdot \hat{\Omega}) \hat{\Omega}
$$

We checked that simple initial spin vectors tracked correctly according to Eq. 11 .

As a test of likely depolarization effects in the neutrino factory due to the differing evolution equations, we examined the polarization in an early example of the double phase rotation front end scenario [13]. This example uses a pion input beam file made from the interactions of 24 $\mathrm{GeV} / \mathrm{c}$ protons on a mercury target. There is a solenoidal field of $20 \mathrm{~T}$ around the target that tapers down to $1.25 \mathrm{~T}$ over a distance of about $4 \mathrm{~m}$ and then continues at that value over the $45 \mathrm{~m}$ long $r f$ phase rotation channel. The actual phase rotation is done with a sequence of $\mathrm{rf}$ cavities that vary in frequency from 30 to $45 \mathrm{MHz}$ and that have electric field gradients up to $6 \mathrm{MV} / \mathrm{m}$. There were no windows or other materials in the muon path in the phase rotation channel. The 
phase rotation is followed by a $1.8 \mathrm{~m}$ long liquid hydrogen minicooler and then a $10 \mathrm{~m}$ long field reversal region where the solenoid field changes from $+1.25 \mathrm{~T}$ to $-1.25 \mathrm{~T}$. The actual details of the channel are not so important here. We are only looking to examine the effects of propagating the muon beam through a long channel containing representative types of electric and magnetic fields.

Fig. 2 shows the behavior of the helicity and spin vector for one particle in the target region. The magnetic field changes very rapidly in this region, starting with a peak value of $20 \mathrm{~T}$ at the target. We see that the spin vector undergoes smooth oscillations through this region and that the helicity is approximately constant.

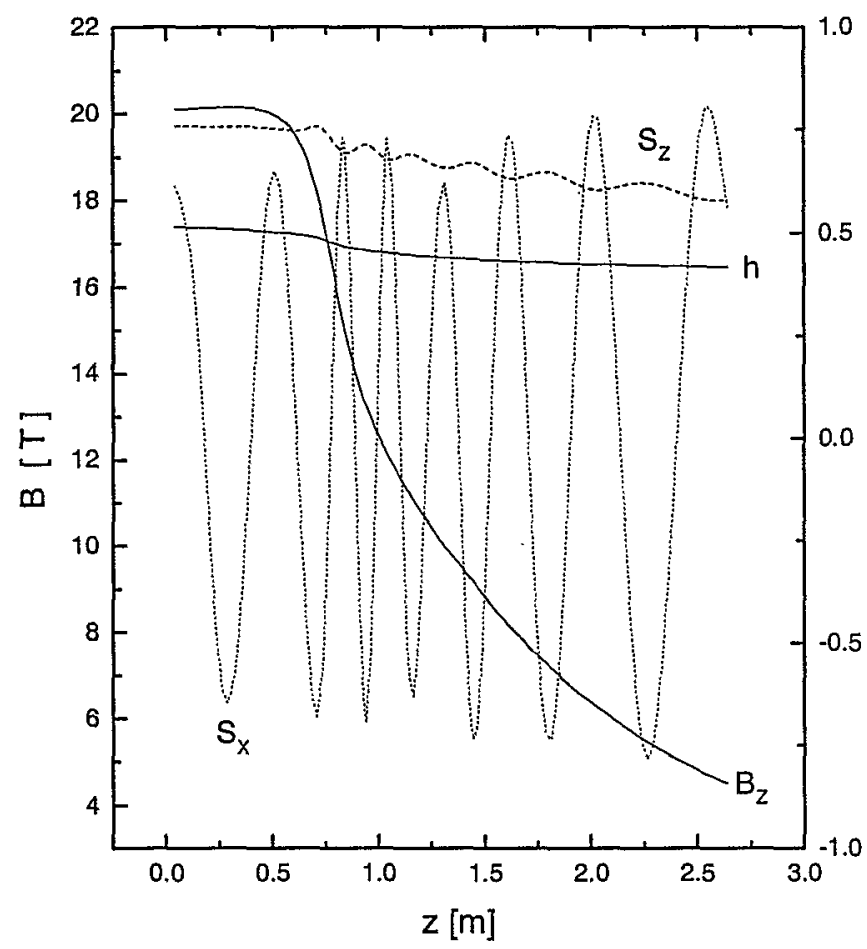

Figure 2. Magnetic field, two spin vector components, and helicity for one particle in the target region. 
Fig. 3 shows the behavior of the spin vector and helicity for one particle at the beginning of the $40 \mathrm{MHZ}$ rf cavities. The spin component motion is regular and the helicity is approximately constant in passing through the $r f$ fields.

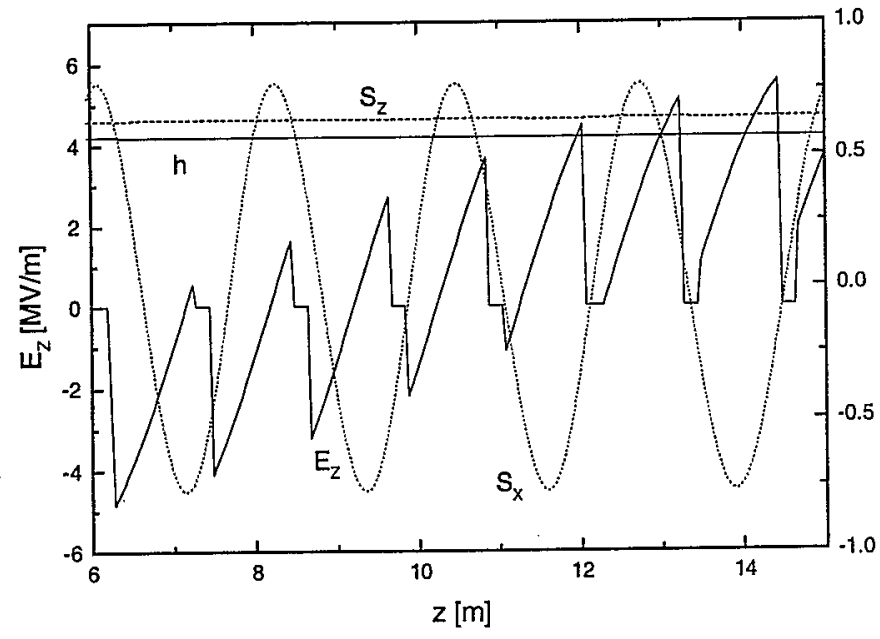

Figure 3. Electric field, two spin vector components, and helicity for one particle in the first $r f$ cavity region. 
Fig. 4 shows the behavior of the spin vector and helicity for one particle in the field reversal region. Again we see that the behavior of the spin components is smooth and that there is little affect on the helicity.

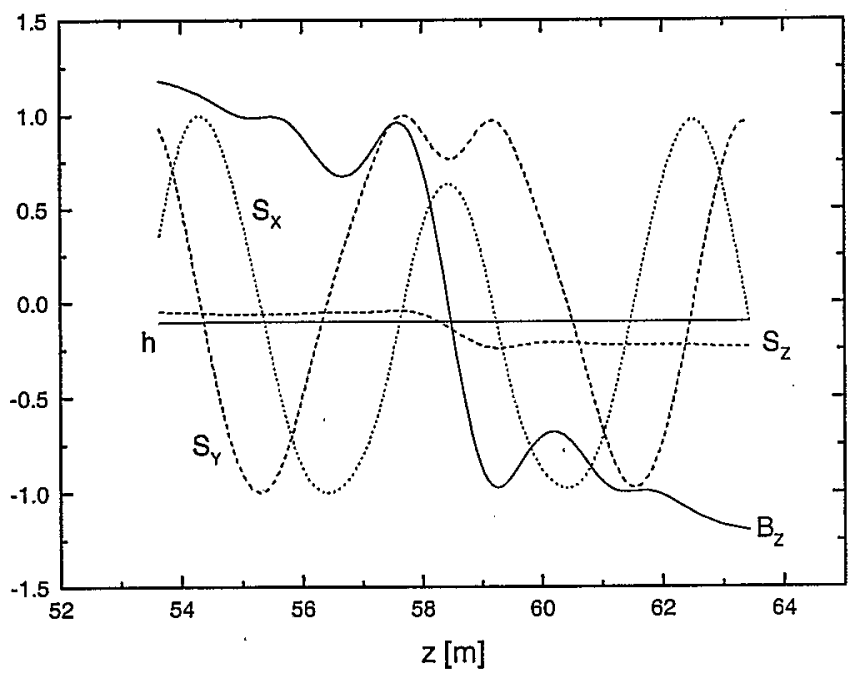

Figure 4. Magnetic field, spin vector components, and helicity for one particle in the first field reversal region. 
When the muons are propagated through the phase rotation system with spin tracking turned off we find that the average helicity of all muons accepted in the channel at the entrance to the minicooler is $-0.189 \pm 0.010$. There is no material in the beam up to this point. The total transmission is $70.5 \%$. With spin tracking enabled the average helicity of the accepted muons drops slightly to $-0.181 \pm 0.010$. A special run with the g-factor anomaly set to 0 gave $-0.188 \pm$ 0.010 , so most of the deviation can be attributed to the extra terms in the spin equation of motion. The distribution of muon helicity at the end of the channel is shown in Fig. 5.

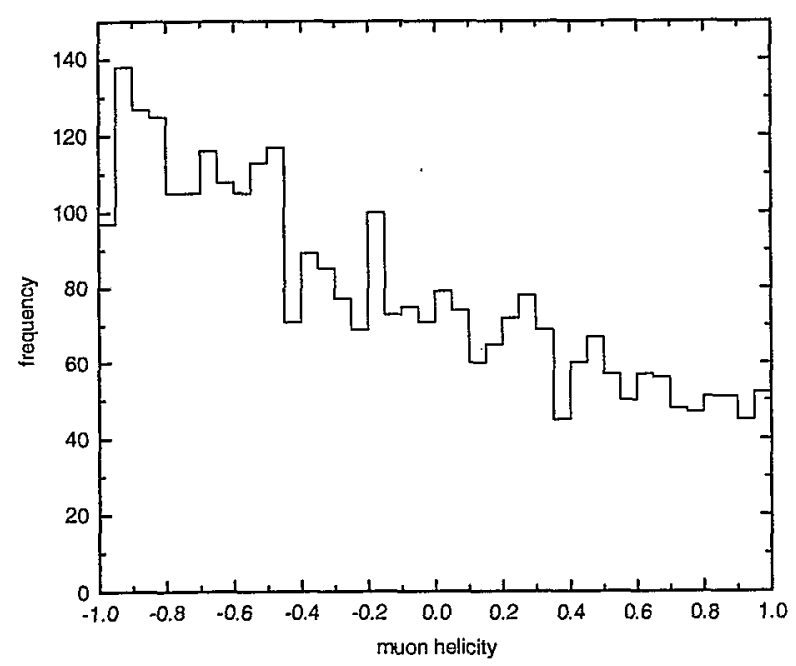

Figure 5. Helicity distribution of all accepted muons at the entrance to the liquid hydrogen minicooler $(z=51.6 \mathrm{~m})$.

The $\sim 4 \%$ depolarization in the channel is comparable to the $\sim 5 \%$ loss found by Andy Van Ginneken [14] in an earlier study of a different phase rotation channel.

\section{Depolarization effects in matter}

Each scattering of a muon in matter has a finite probability of changing the muon's spin direction. This in turn can lead to a depolarization of the muon beam. We have considered two possible depolarizing processes, elastic and multiple Coulomb scattering. Both of these processes change the momentum vector of the muon. Our approach is to first change the spin vector in each interaction in such a way that the helicity is conserved. Then, using the appropriate kinematics for the event, we determine the quantum mechanical probability for the helicity to flip in the interaction. We then randomly flip muon spin directions with that probability. 
Continuous energy loss (Bethe-Bloch) is another important process that is simulated for particles in matter. However, in the continuous energy loss process the code scales the magnitude of the momentum vector to correspond to the reduced kinetic energy available after a step. The direction of the momentum is unchanged in this process, and thus, according to Eq. 1, the helicity is not changed by it.

The helicity is forced to be conserved in scattering events by first computing the helicity $h$ before each scattering. Then, if primes refer to quantities after the scattering, the spin vector after the scattering is taken as

$$
\hat{s}^{\prime}=h \hat{p}^{\prime}+\sqrt{1-h^{2}} \frac{\left(\hat{p}^{\prime} \times \hat{s}\right) \times \hat{p}^{\prime}}{\left|\left(\hat{p}^{\prime} \times \hat{s}\right) \times \hat{p}^{\prime}\right|}
$$

As a test of the effectiveness of this algorithm, we show in Fig. 6 the behavior of the helicity in the $1.8 \mathrm{~m}$ long liquid hydrogen minicooler. The helicity remains approximately constant, even though numerous scattering events occur and the kinetic energy drops by a large amount.

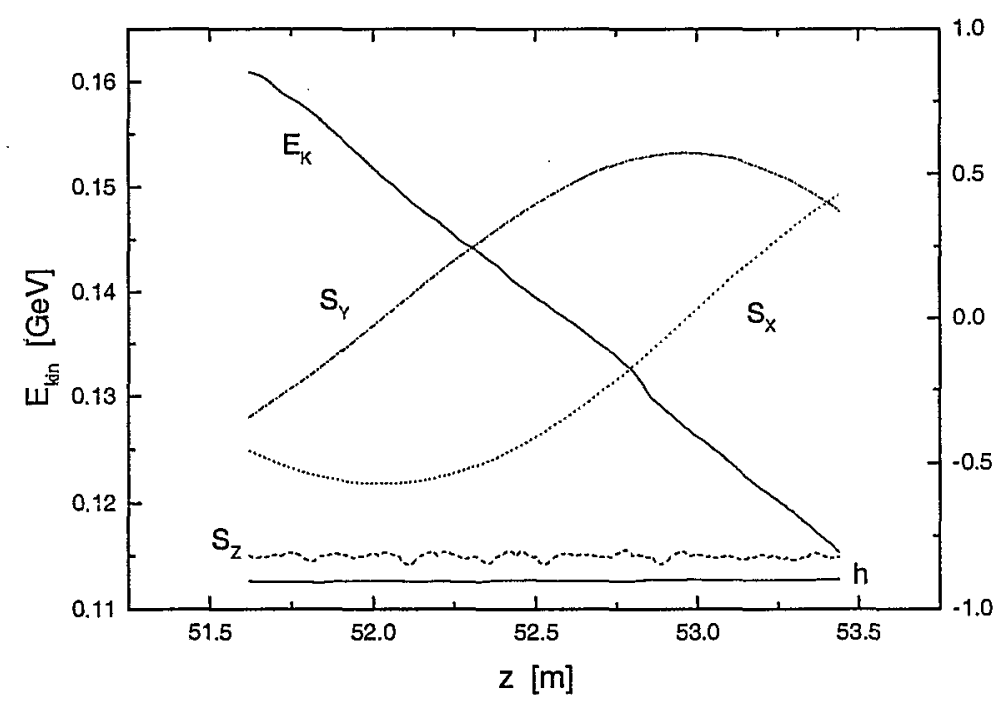

Figure 6. Kinetic energy, spin vector components, and helicity for one particle in the liquid hydrogen minicooler.

Norum \& Rossmanith [15] have given an estimate of the spin flip probability 


$$
P r_{\text {flip }} \approx \frac{m_{e}}{m_{\mu}} \beta^{2} \frac{\Delta E}{E}
$$

where $\Delta \mathrm{E} / \mathrm{E}$ is the fractional energy loss in the material. This expression is based on relating the energy loss to the scattering angle and using the non-relativistic calculation of the spin flip probability of Ford \& Mullin, described below.

The calculated spin flip probability in multiple Coulomb scattering is known to be very small [16-19]. The spin flip probability, obtained by integrating the $1^{\text {st }}$ order QED matrix element [18] over scattering angles, is

$$
\operatorname{Pr}_{m c s}=h d K J L \ln (M)
$$

where $h$ is the helicity and $d$ is the current step size. The quantities $\mathrm{K}, \mathrm{J}, \mathrm{L}$ and $\mathrm{M}$ are given by

$$
\begin{aligned}
K & =\pi r_{e}^{2} N_{A} m_{e}^{2} c^{4} m_{\mu}^{2} c^{4} \\
J & =\frac{\rho Z^{2}}{A} \\
L & =\frac{1}{(\beta p E)^{2}} \\
M & =\frac{1-\cos \left(\frac{2}{\left.\alpha A^{\frac{1}{3}} \frac{m_{e} c}{p}\right)}\right.}{1-\cos \left(\alpha Z^{\frac{1}{3}} \frac{m_{e} c}{p}\right)}
\end{aligned}
$$

For typical step size $\sim 1 \mathrm{~mm}$ in liquid hydrogen this probability is less than $10^{-7}$ and is thus indeed negligible. 
Ford \& Mullin [19] have computed the non-relativistic spin flip probability in elastic scattering as a function of the muon scattering angle. The probability that a muon that is scattered through the CM angle $\theta^{*}$ ends up with its spin in the opposite direction from the direction before the scattering is

$$
\begin{aligned}
\operatorname{Pr}_{f l i p} & =\frac{m_{e}^{2}}{m_{\mu}^{2}} \beta^{4}\left(\sin ^{2} \frac{\theta^{*}}{2}-\sin ^{4} \frac{\theta^{*}}{2}+\sin ^{6} \frac{\theta^{*}}{2}\right) \\
& =\frac{m_{e}^{2}}{m_{\mu}^{2}} \frac{\beta^{4}}{8}\left(-\cos ^{3} \theta^{*}+\cos ^{2} \theta^{*}-3 \cos \theta^{*}+3\right)
\end{aligned}
$$

This expression is plotted in Fig. 7.

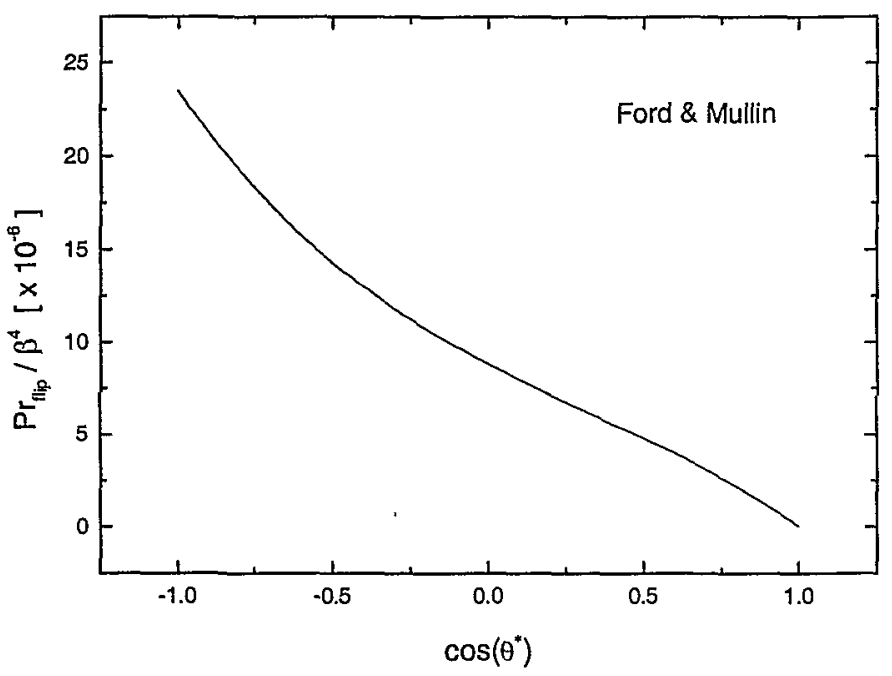

Figure 7. Probability that a muon scattered elastically through an angle $\theta^{*}$ ends up with its spin in the opposite direction from the direction before the scattering. Calculation done by Ford \& Mullin.

Note that the probability for spin flip is 0 in the forward direction. The probabilities are small in all cases, in agreement with the general principle that in non-relativistic scattering the particle's spin tends to remain aligned with its initial direction. 
We have recently extended these calculations [16] by computing the lowest order QED matrix element for the helicity flip probability in elastic scattering. The results are shown in Fig. 8 for several values of the muon LAB momentum.

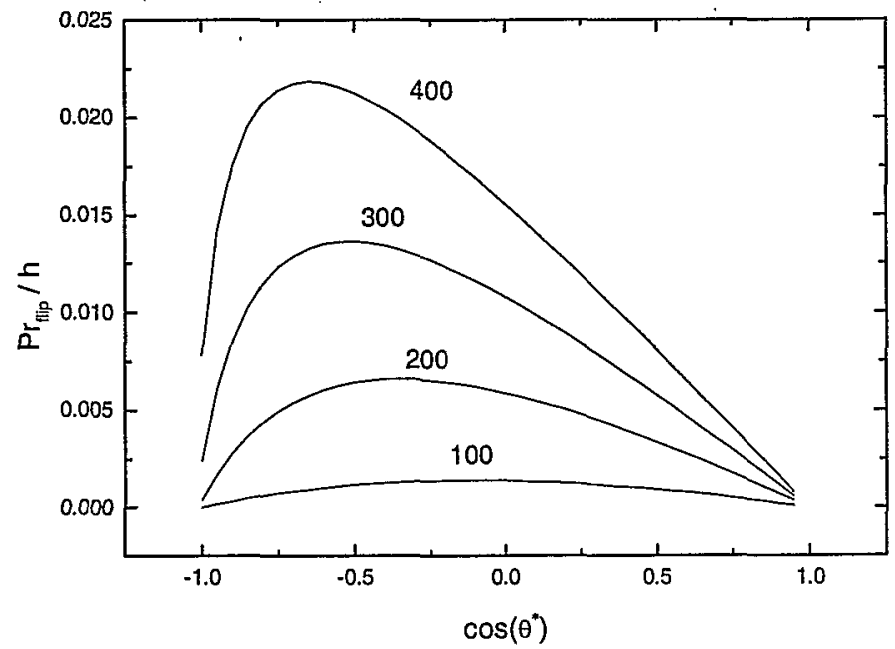

Figure 8. First order QED calculation of the probability that a muon scattered elastically through an angle $\theta^{*}$ ends up with its spin in the opposite direction from the direction before the scattering. The probability is divided by the particle's helicity. The numbers on the curves indicate the muon LAB momentum in $\mathrm{MeV} / \mathrm{c}$.

The spin flip probability vanishes in both the forward and backwards directions. For muons with a typical momentum of $200 \mathrm{MeV} / \mathrm{c}$, the maximum spin flip probability in an elastic scattering event is $\sim 510^{-3}$. 


\section{Conclusions}

We feel that the data presented here shows that the current version of Icool (2.06) can in principle simulate spin effects in the front end of a neutrino factory. We have done an initial test of the code by simulating the full $480 \mathrm{~m}$ long, double phase rotation system described in reference [13]. Figure 9 shows the average helicity in each bunch at the end of the system. We see that the average bunch helicity varies from about -60 to $+60 \%$. There is a strong correlation of the strength of the helicity with its temporal location in the bunch train. However, the average helicity is not a meaningful measure for the distribution as a whole. We define instead the effective polarization

$$
P_{\text {eff }}=\int h^{2}(t) I(t) d t
$$

where $I(t)$ is the normalized intensity at time $t$. The effective polarization was $0.355 \pm 0.007$ at 52 $m$ into the channel (at the entrance to the minicooler, after the decay region). The effective polarization at the end of the channel was $0.350 \pm 0.015$. Thus the loss in effective polarization was only $1.4 \%$, although we caution again that these are the first physics results obtained with the code.

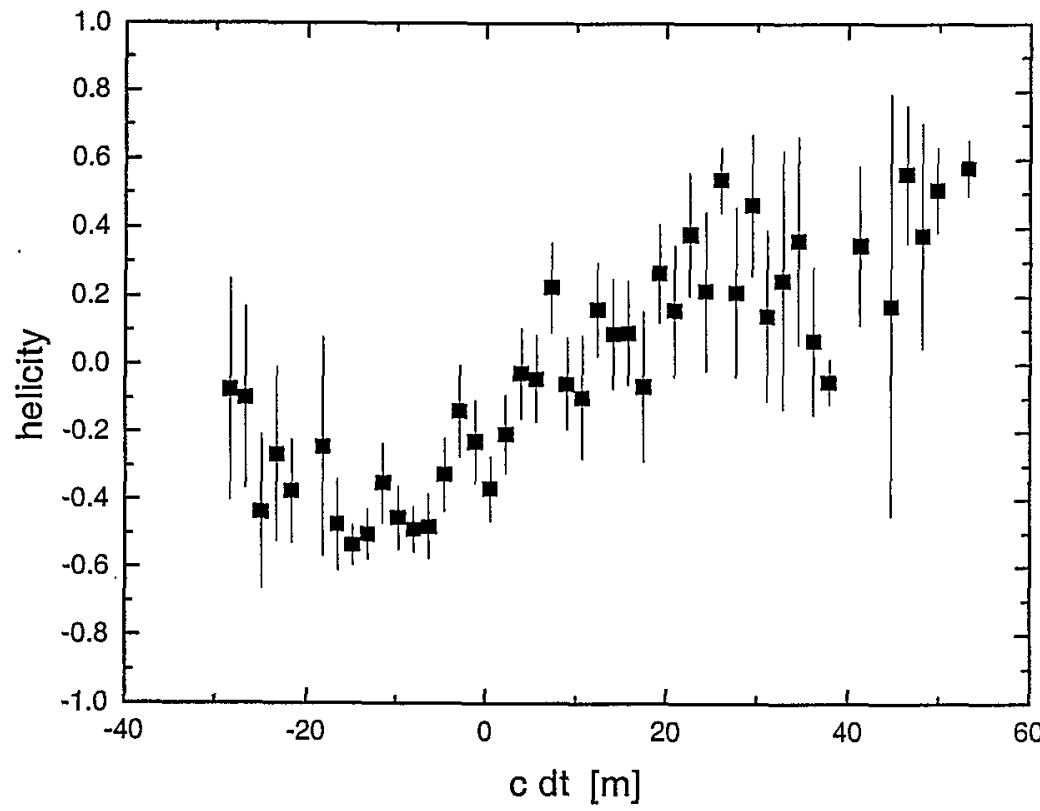

Figure 9. Correlation of average helicity with bunch position at the end of the double phase rotation system. 


\section{Acknowledgments}

We would like to thank Alain Blondel, Andy van Ginneken, and Bob Palmer for useful discussions.

\section{Appendix: Analytic calculation of the asymptotic helicity}

We now derive an analytic expression for the asymptotic value of the muon helicity in the LAB frame. For any given pion momentum in the $\mathrm{LAB}$ there are a range of muon helicities possible, corresponding to production of the muon at different center of mass angles $\theta^{*}$ in the pion rest frame. Since the pion has spin 0 , the muons have an isotropic distribution in angle in the pion rest frame. Let us define $x=\cos \theta^{*}$. Then the average helicity corresponding to a given pion momentum is

$$
<h>=\frac{1}{2} \int_{-1}^{1} h(x) d x
$$

Using Eq. 3 for $h(x)$, we have

$$
<h>=\frac{1}{2} \int_{-1}^{1}\left[\frac{E_{\pi} p^{*}+E^{*} p_{\pi} x}{m_{\pi} p(x)}\right] d x
$$

If we take the $\mathrm{z}$ axis to lie along the pion direction and compute the components of the muon $\mathrm{LAB}$ momentum parallel and perpendicular to $\mathrm{z}$, we find that

$$
p(x)=\frac{1}{m_{\pi}}\left[p^{* 2} m_{\pi}^{2}+p_{\pi}^{2} E^{* 2}+2 p^{*} p_{\pi} E^{*} E_{\pi} x+E_{\pi}^{2} p^{* 2} x^{2}-p^{* 2} m_{\pi}^{2} x^{2}\right]^{\frac{1}{2}}
$$

Substituting into Eq. 20, we obtain the integral

$$
<h>=\int_{-1}^{1} \frac{\alpha+\beta x}{\sqrt{R(x)}} d x
$$


where

$$
\begin{aligned}
R(x) & =a+b x+c x^{2} \\
a & =p^{* 2}+\frac{p_{\pi}^{2} E^{* 2}}{m_{\pi}^{2}} \\
b & =\frac{2 p^{*} p_{\pi} E^{*} E_{\pi}}{m_{\pi}^{2}} \\
c & =\frac{p^{* 2} p_{\pi}^{2}}{m_{\pi}^{2}} \\
\alpha & =\frac{E_{\pi} p^{*}}{2 m_{\pi}} \\
\beta & =\frac{p_{\pi} E^{*}}{2 m_{\pi}}
\end{aligned}
$$

The integral can now be performed using standard forms in the tables [20] as

$$
\begin{aligned}
<h> & =\left(\alpha-\frac{\beta b}{2 c}\right) \frac{1}{\sqrt{c}} \ln \left[\frac{2 \sqrt{c R_{+}}+2 c+b}{2 \sqrt{c R_{-}}-2 c+b}\right] \\
& +\frac{\beta}{c}\left(\sqrt{R_{+}}-\sqrt{R_{-}}\right)
\end{aligned}
$$

where

$$
\begin{aligned}
& R_{+}=a+b+c \\
& R_{-}=a-b+c
\end{aligned}
$$

If we now take the limit of this equation as $\beta_{\pi}$ approaches 1 , we obtain an expression

$$
<h>=\frac{1}{2}\left(1-\frac{E^{* 2}}{p^{* 2}}\right) \ln \left[\frac{E^{*}+p^{*}}{E^{*}-p^{*}}\right]+\frac{E^{*}}{p^{*}}
$$

defined entirely in terms of the muon kinematics in the pion rest frame. Using $\mathrm{p}^{*}=30 \mathrm{MeV}$ and $\mathrm{E}^{*}=110 \mathrm{MeV}$, we find that $\langle\mathrm{h}\rangle=0.185$, in agreement with the simulations. 


\section{Notes and references}

[1] A. Blondel, Muon collider collaboration meeting, LBNL, December, 1999; http:/aleph.www.cern.ch/ bdl/muon/nufacpol

[2] W. Marciano, The physics of muon colliders: a perspective, in D. Cline (ed), Physics Potential and Development of $\mu+\mu$-Colliders, AIP Conf. Proc. 441, 1998, p.347-53.

[3] D. Perkins, Introduction to High Energy Physics, Addison-Wesley, 1972, p.150.

[4] S. Hayakawa, Polarization of cosmic ray $\mu$ mesons: theory, Phys. Rev. 108:1533-7, 1957.

[5] M. Perl, High Energy Hadron Physics, Wiley, 1974, p.232.

[6] J. Gallardo, R. Fernow \& Y. Fukui, A short note on the Wigner rotation, BNL Muon Tech Note MU-039, 2000.

[7] J.D. Jackson, Classical Electrodynamics, $3^{\text {rd }}$ ed., Wiley, p. 561-5.

[8] The Hayakawa, Wigner, and Jackson expressions are all computed in the subroutine Decay in Icool and listed in the diagnostic print out. The Hawakaya equation is used for histograms, statistical summaries and saved in post processor output.

[9] The $\mu+\mu$-Collider Collaboration, Muon-muon collider feasibility study, in New Directions for High Energy Physics, Proc. Snowmass 96, p. 432; C. Ankenbrandt et al, Status of muon collider research and development and future plans, Phys. Rev. ST-AB 2:081001-1-73, 1999, p. 20.

[10] S. Hayakawa, ref 4,. eqs. 8, 4 , and 9.

[11] J. Jackson, ref. 7, eq. 11.158 .

[12] S.Y. Lee, Spin Dynamics and Snakes in Synchrotrons, World Scientific, 1997, p.9-14.

[13] The case we considered was the PJK scenario taken from

http://pubweb.bnl.gov/people/palmer/nu/pjksim/icool/

as the data files existed on 25 March 2000.

[14] A. Van Ginneken, Polarization of muons in decay channel, Mucool note \#65, 1999.

[15] B. Norum \& R. Rossmanith, Polarized beams in a muon collider, Nuc. Phys. B (Proc.

Suppl.) 51A:191-200, 1996.

[16] R. Fernow, J. Gallardo \& Y. Fukui, On muon depolarization effects in an ionization cooling channel, NuMu note 115, 2000.

[17] M. Rose \& H. Bethe, On the absence of polarization in electron scattering, Phys. Rev. 55:277-289, 1939

[18] J.D. Bjorken \& S.D. Drell, Relativistic Quantum Mechanics, McGraw-Hill, 1964, eq. 7.99.

[19] G. Ford \& C. Mullin, Scattering of polarized Dirac particles on electrons, Phys. Rev.

108:477-481, 1957.

[20] I. Gradshteyn \& I. Ryzhik, Tables of Integrals, Series and Products, Academic Press, 1980, p.81. 